GP53 ELUSIVE CAUSE FOR A RECURRENT FEVER IN A 2-YEAROLD BOY

Doris Burnaz*, Simona Tătar. Emergency Pediatric Hospital, Third Pediatric Clinic, ClujNapoca, Romania

\subsection{6/archdischild-2019-epa.119}

Introduction Periodic fever syndromes (PFSs) can present with a myriad of nonspecific signs and symptoms, with acute onset fever as the hallmark.

Case description We present the case of a 2-year-old boy who was admitted on 3 occasions with episodes of sudden onset isolated fever $\left(40^{\circ} \mathrm{C}\right)$. From his history we noted 2 more hospital admittances in another medical center with similar presentations; also, the mother reported 4 more isolated fever episodes, the first at 4 months of age, during which the fever was apparently uninfluenced by antipyretics and disappeared after 3-5 days (no inflammatory markers were taken then as he was treated as an outpatient). A detailed history showed a clear periodicity of the episodes, occurring at 28-30 days intervals. Other symptoms were intermittently present during episodes: macular rash, unilateral cervical adenopathy $(2 \mathrm{~cm})$. Fever duration was 3 to 5 days, without a specific response to antipyretic or antibiotic treatment. A slight response to oral prednisone therapy was noted. At each admission, preliminary laboratory studies showed a marked inflammatory response (high CRP, procalcitonin, VSH, WBC). Broad spectrum antibiotic treatment was promptly started after blood and urine were sampled for cultures, as the presentations were interpreted at that time as high suspicion of occult sepsis/bacteremia. All sources of infection were ruled out each time by negative cultures. Blood smears and imaging studies did not raise any suspicion of malignancy. Tests for a primary immunologic defect were negative. PFSs were partially excluded as ANA titers, rheumatoid factor, IgD level was normal (HIDS), urinary mevalonic acid (MKD) was undetectable. Also, a genetic panel including 32 gene mutations/variations associated with PFSs including Familial Mediterranean Fever, CAPS, TRAPS, Blau syndrome, HIDS, ELANE related neutropenia and PAPA, did not detect mutations. All inflammatory markers returned to normal in between fever episodes. Our patient developed 3 more similar episodes, then the symptoms spontaneously stopped. The child is presently healthy, 6 months apart from the last episode.

Discussion PFSs are a diagnostic challenge. The key to a correct clinical approach is close monitoring of episodes and extensive workup. It seems that some causes remain undetermined despite diagnostic efforts or, as in our case, have a self-limited evolution. Future research in this pathology is still necessary.

\section{GP54 SLEEPING SICKNESS: CONGENITAL CASE ASSOCIATED WITH A POSSIBLE SEXUAL TRANSMISSION}

${ }^{1}$ Ana Maia* , ${ }^{1}$ Ana Rita Curval, ${ }^{1}$ Tiago Magalhães, ${ }^{1}$ Micaela Guardiano, 1,2 Inês Azevedo, ${ }^{3}$ Jorge Seixas, ${ }^{3}$ Jorge Atouguia. ${ }^{1}$ Centro Materno Pediátrico, Centro Hospitalar Universitário São João, Porto, Portugal; ' ${ }^{2}$ aculdade de Medicina da Universidade do Porto, Porto, Portugal; ${ }^{3}$ Instituto de Higiene e Medicina Tropical, Lisboa, Portugal

10.1136/archdischild-2019-epa.120

Human African trypanosomiasis (HAT), also known as sleeping sickness, is caused by protozoan parasites transmitted by the bite of a tsetse fly. It is characterized by an early stage, during which trypanosomes circulate in the blood or lymphatics, and a late stage, in which there is involvement of the central nervous system. In a globalised world, some cases are also diagnosed outside endemic African countries, thus HAT should be considered in differential diagnosis for travellers, tourists and migrants.

The authors report a rare case of a 19-year-old Afro-Brazilian boy, born in the USA, who was diagnosed with a congenital infection by Trypanosoma brucei gambiense at 16 months of age after returning to Portugal. Neither the patient nor his mother had ever been to Africa. He was admitted in our Paediatric Department for investigation of a wasting syndrome, lymphadenopathies and intermittent fever. An initial evaluation showed anaemia, hypergammaglobulinemia and a positive serology for cytomegalovirus infection. A month later the wasting syndrome had become worse and daytime somnolence was identified. At this point, the diagnosis of HAT was established in his mother, motivating specific diagnostic procedures in the child. Trypanosomes were detected in his blood and a CATT (Card Agglutination Test for Trypanosomes) was positive. A lumbar puncture established the diagnosis of late stage HAT. Electroencephalogram and brain MRI showed extensive brain damage. Fundoscopic examination revealed spots of an atypical chorioretinitis, possibly in association with the combined trypanosomal and cytomegalovirus infection. Treatment was established with intravenous eflornithine (DFMO). The drug was well tolerated, resulting in a gradual clinical improvement, including the complete regression of the brain and retinal changes. Two years after DFMO administration parasitological cure was confirmed according to established criteria. At the moment, the patient remains well, and show a normal psychomotor development.

The absence of suggestive epidemiological data in our patient and his mother made it difficult to establish the correct diagnosis. The correct diagnosis of our patient's condition was possible only after the etiology of his mother's disease was correctly established in our hospital. Given the available epidemiological data, transmission of HAT between our patient's parents must have occurred during sexual intercourse and is the first description of a case of probable sexual transmission of the disease. In our case, since neither the mother nor her son had ever been to Africa, the congenital transmission of HAT is indisputable.

\section{GP55 GOING OUT ON A (CLOTTED) LIMB - AN INTERESTING CASE OF PAGET-SCHROETTER SYNDROME IN A YOUNG ADOLESCENT}

${ }^{1}$ Therese Martin*, ${ }^{1}$ Mary Moore, ${ }^{1}$ Laura Melody, ${ }^{2}$ Beatrice Nolan, ${ }^{3}$ Aisling Snow, ${ }^{4}$ Pradeep Govender, ${ }^{1}$ Carol Blackburn. 'Department of Emergnecy Medicine, Our Lady's Children's Hospital Crumlin, Dublin, Ireland; 'Department of Haematology, Our Lady's Children's Hospital Crumlin, Dublin, Ireland; 'Department of Radiology, Our Lady's Children's Hospital Crumlin, Dublin, Ireland; ${ }^{4}$ Department of Interventional Radiology, Tallaght University Hospital, Dublin, Ireland

\subsection{6/archdischild-2019-epa.121}

Introduction A 13-year-old female, presented to the Paediatric Emergency Department (PED), with a 4 week history of bruising to her right upper limb. There was no concurrent history of trauma to the limb. Bruising was progressive, and the arm had become increasingly painful in the three days prior to 